Article

\title{
Quantitative Determination of the Surface Distribution of Supported Metal Nanoparticles: A Laser Ablation-ICP-MS Based Approach
}

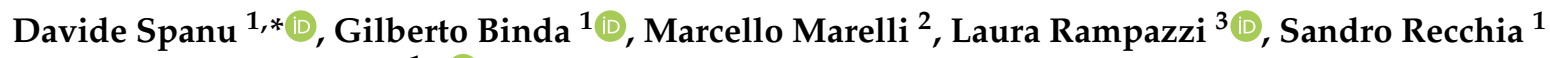 \\ and Damiano Monticelli ${ }^{1, *(\mathbb{D})}$ \\ 1 Department of Science and High Technology, University of Insubria, Via Valleggio, 11, 21100 Como, Italy; \\ gilberto.binda@uninsubria.it (G.B.); sandro.recchia@uninsubria.it (S.R.) \\ 2 National Research Council of Italy (CNR), Istituto SCITEC, Via C. Golgi 19, 20133 Milano, Italy; \\ marcello.marelli@scitec.cnr.it \\ 3 Department of Human Sciences and Innovation for the Territory, University of Insubria, \\ via Sant'Abbondio 12, 22100 Como, Italy; laura.rampazzi@uninsubria.it \\ * Correspondence: d.spanu1@uninsubria.it (D.S.); damiano.monticelli@uninsubria.it (D.M.); \\ Tel.: +39-0312386451 (D.S.); +39-0312386427 (D.M.)
}

\section{check for}

updates

Citation: Spanu, D.; Binda, G.; Marelli, M.; Rampazzi, L.; Recchia, S.; Monticelli, D. Quantitative

Determination of the Surface Distribution of Supported Metal Nanoparticles: A Laser Ablation-ICP-MS Based Approach Chemosensors 2021, 9, 77.

https://doi.org/10.3390/

chemosensors 9040077

Academic Editor: Vardan Galstyan

Received: 26 February 2021

Accepted: 8 April 2021

Published: 10 April 2021

Publisher's Note: MDPI stays neutra with regard to jurisdictional claims in published maps and institutional affiliations.

Copyright: (c) 2021 by the authors. Licensee MDPI, Basel, Switzerland. This article is an open access article distributed under the terms and conditions of the Creative Commons Attribution (CC BY) license (https:/ / creativecommons.org/licenses/by/ $4.0 /)$.

\begin{abstract}
A laser ablation-inductively coupled plasma-mass spectrometry (LA-ICP-MS) based method is proposed for the quantitative determination of the spatial distribution of metal nanoparticles (NPs) supported on planar substrates. The surface is sampled using tailored ablation patterns and the data are used to define three-dimensional functions describing the spatial distribution of NPs. The volume integrals of such interpolated surfaces are calibrated to obtain the mass distribution of Ag NPs by correlation with the total mass of metal as determined by metal extraction and ICP-MS analysis. Once this mass calibration is carried out on a sacrificial sample, quantifications can be performed over multiple samples by a simple micro-destructive LA-ICP-MS analysis without requiring the extraction/dissolution of metal NPs. The proposed approach is here tested using a model sample consisting of a low-density polyethylene (LDPE) disk decorated with silver NPs, achieving high spatial resolution over $\mathrm{cm}^{2}$-sized samples and very high sensitivity. The developed method is accordingly a useful analytical tool for applications requiring both the total mass and the spatial distribution of metal NPs to be determined without damaging the sample surface (e.g., composite functional materials and NPs, decorated catalysts or electrodic materials).
\end{abstract}

Keywords: laser ablation; ICP-MS; LA-ICP-MS; metal nanoparticles; metal deposition; calibration; silver; supersonic cluster beam deposition; trace elements

\section{Introduction}

The deposition of metal nanoparticles (NPs) on the surface of solid supports is an increasingly used strategy to produce functional materials for many applications including catalysis [1-3], sensing devices [4-6] and electronics [7,8]. A wide variety of deposition techniques, such as sputtering $[9,10]$, pulsed laser deposition $[11,12]$, photo-deposition $[13,14]$, electro-deposition $[15,16]$, precipitation $[17,18]$ and drop-casting $[19,20]$ are described in the literature. The variety of metal deposition strategies leads to a great difference in the mass, size, geometry and spatial distribution of deposited NPs. Moreover, the experimental conditions (pressure and temperature) and the nature of the substrate (its morphology and wettability) have a noticeable effect on NP deposition.

Most of the above-mentioned strategies ideally lead to the homogeneous deposition of metal NPs [21], but physical sputtering and laser-induced direct deposition can generate non-homogeneous NP distributions [22,23], especially over large-sized supports. Variations in NPs' spatial loading are often intentionally induced, as they can positively affect optical and electronic properties of the composite material and, consequently, its 
performances for electrochemical and catalytic applications [23-25]. Analytical tools to quantitatively determine the spatial distribution of NPs over a given substrate are accordingly highly desirable.

These investigations are commonly based on qualitative morphological observations by scanning electron microscopy (SEM). The latter has the advantage to be non-destructive, but it is extremely time-consuming and requires an unpractical very high number of images to be acquired when medium or large sized samples must be studied. Information about the elemental distribution may be achieved by coupling an energy dispersive Xray spectrometer with SEM (SEM-EDS), but the analyzable sample size is again strongly limited. In any case, EDS analysis is totally inappropriate to perform quantitative analyses for two main reasons: (i) it is quite difficult to obtain reliable calibrations in the form signal vs. absolute quantity; (ii) the poor detection limits of EDS (around $0.1 \%-0.5 \% \mathrm{wt}$.) makes the detection of limited amounts of NPs very difficult, even if they are clearly visible in SEM images [26]. Summing up, SEM-based analysis provides indications only on the local NPs' density over microscopic portions of highly loaded samples.

As opposite, extraction/dissolution and acid assisted digestions followed by quantification by sensitive instrumental methods are commonly employed to gain useful information even for very low metal loadings $[27,28]$. Nevertheless, this approach is clearly destructive and it provides no information about the NPs' spatial distribution. Alternatively (but more rarely), mass sensitive piezoelectric devices, such as quartz crystal microbalances, can be employed to monitor down to nanograms of loaded metal when vacuum deposition techniques are used [29,30]. In this case, the integrity of the sample is preserved, but information regarding the spatial distribution of metal NPs is still missing.

Laser ablation-inductively coupled plasma-mass spectrometry (LA-ICP-MS) seems a promising candidate to quantitatively investigate NPs' spatial distribution. LA-ICP-MS is an increasingly employed method for the direct analysis of trace elements in/on solid materials and finds application in many research fields, e.g., biology [31], environmental science [32], dendrochemistry [33], medicine [32], geoscience [34] and cultural heritage [35]. Recent advancements in the design of LA-ICP-MS instrumentation made possible the achievement of very good spatial resolution and high sensitivity, enabling the detection of trace elements over surfaces tens of square centimeters in size [36,37]. Besides these beneficial analytical features, the micro-sized laser spot used for these measurements causes very limited damage to the sample as only a negligible part of the surface is ablated. Owing to its high performances, LA-ICP-MS also found application for both morphological and quantitative analysis of NP deposited on various substrates [38,39]. Single particle analysis [39] and the application of micro-dried droplets for quantitative analysis [38] currently represent two major advancements in the field. Although LA-ICP-MS has aroused great interest in the field of NPs, the quantitative determination of their spatial distribution on planar substrates is still a challenge never faced using this technique.

In view of these considerations, we developed an LA-ICP-MS analytical protocol to generate a quantitative distribution map of metal NPs deposited over planar substrates, by selecting the proper sampling patterns and data elaboration. The proposed approach is tested for the first time using a model sample consisting of silver NPs anisotropically distributed over low-density polyethylene (LDPE) disks.

\section{Materials and Methods}

\subsection{Samples Preparation and Characterization}

A pulsed microplasma cluster source (PMCS) was used to perform a supersonic cluster beam deposition (SCBD) of Ag NPs over LDPE disks ( $\sim 2 \mathrm{~cm}$ diameter). The instrumental system is schematically shown in Figure 1, and described in detail elsewhere [40-42]. Briefly, a PMCS consists of an anodic cavity where an Ag rotating rod cathode is inserted: the introduction of triggered pulses of Ar gas ( $45 \mathrm{bar}$ for $370 \mu \mathrm{s}$ ) and high voltages pulses ( $900 \mathrm{~V}$ for $120 \mu \mathrm{s}$, with a $500 \mathrm{~ms}$ delay with respect to the end of the Ar pulse) leads to the formation of a pulsed plasma $(3 \mathrm{~Hz})$ and, consequently, to the pulsed emission of $\mathrm{Ag}$ 
atoms which thermalize with the carrier gas and aggregate in the cavity, forming thus small metal clusters. The carrier gas-cluster mixture expands out of the PMCS through a series of three aerodynamic lenses into a low-pressure deposition chamber. Due to the highpressure difference between the cavity and the deposition chamber a highly collimated supersonic beam (divergence $<1^{\circ}$ ) of $\mathrm{Ag}$ clusters is generated. In these conditions, all $\mathrm{Ag}$ NPs are implanted in the center of the target. The introduction of a partial pressure of Ar in the deposition chamber allows partially deviated Ag NPs, inducing a more radially spread deposition.

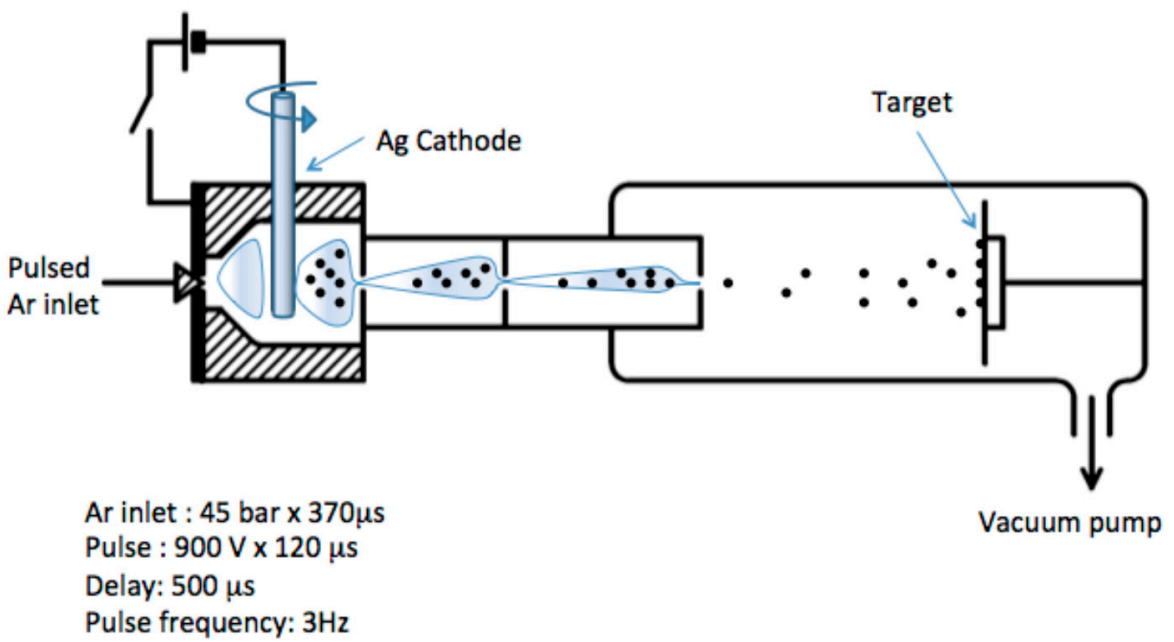

Figure 1. Schematic representation of the employed pulsed microplasma cluster source-supersonic cluster beam deposition (PMCS-SCBD) system used for Ag NP deposition on LDPE disks.

Two types of sample were prepared by varying the deposition time: " $10 s$ " and " $5 \mathrm{~s}$ ", i.e., using deposition times of $10 \mathrm{~s}$ (30 pulses) and $5 \mathrm{~s}$ (15 pulses), respectively.

Samples analyzed right after metal deposition were named "10 s as-deposited" and " $5 \mathrm{~s}$ as-deposited".

One " $5 \mathrm{~s}$ " sample was treated with dichloro-methane vapors $(293 \mathrm{~K}, 47.1 \mathrm{kPa})$ for $4 \mathrm{~h}$ after Ag NPs deposition. This sample is labeled "5 s incorporated".

The morphology of the polymeric disks and Ag NPs was studied using an environmental scanning electron microscope (ESEM FEG XL30, Philips, Eindhoven, The Netherlands) at $20 \mathrm{kV}$ under low vacuum conditions (1.4 torr of water vapor).

Transmission electron microscopy (TEM) and high-resolution TEM (HR-TEM) analysis were performed by a ZEISS LIBRA 200FE microscope. A suitable specimen was prepared by gently scratching the sample surface with a scalpel and collecting the resulting material by simple adherence onto a holey-carbon supported film TEM grid.

The particle-size distribution of silver nanoparticles was determined by processing SEM and TEM images using the software ImageJ (National Institutes of Health (NIH), Bethesda, MD, USA).

\subsection{LA-ICP-MS Analysis}

An inductively coupled plasma-mass spectrometer (ICP-MS), model Thermo Scientific ICAP Q, coupled with a fourth harmonic Nd: YAG $266 \mathrm{~nm}$ laser system, model New Wave UP Series 266, was used for the laser ablation-ICP-MS (LA-ICP-MS) measurements.

A viscous film sample chamber was used for all the LA-ICP-MS measurements (see $[36,43]$ for details).

Ag-loaded polymeric disks were analyzed using both lines and grids of spots (the sampling patterns will be discussed in the Results and Discussion section) employing the operating parameters summarized in Table 1 . The number of laser shots $(20$ within $10 \mathrm{~s})$ was optimized to obtain the total removal of Ag NPs. Peaks resulting from each 
ablation spot were integrated. Such integral values were used to create a map of the metal distribution over the surface of the sample.

Table 1. Operating laser and ICP-MS parameters used for all the LA-ICP-MS measurements.

\begin{tabular}{cc}
\hline & Laser Parameters \\
\hline Laser frequency $(\mathrm{Hz})$ & 2 \\
Spot diameter $(\mu \mathrm{m})$ & 5 (round-shaped) \\
Laser fluence $\left(\mathrm{J} / \mathrm{cm}^{2}\right)$ & 1.35 \\
Dwell time for each spot $(\mathrm{s})$ & 10 \\
Delay spot-by-spot $(\mathrm{s})$ & 5 \\
Helium flux $(\mathrm{L} / \mathrm{min})$ & 0.85 \\
\hline \multicolumn{1}{c}{} & \\
\hline RF power $(\mathrm{kW})$ & 1.55 \\
Auxiliary gas flux $(\mathrm{L} / \mathrm{min})$ & 0.8 \\
Cooling gas flux $(\mathrm{L} / \mathrm{min})$ & 14 \\
Makeup gas flux $(\mathrm{L} / \mathrm{min})$ & 0.9 \\
Dwell time $(\mathrm{ms})$ & 10 \\
$m / z$ channel & $107 \mathrm{Ag}$ \\
\hline
\end{tabular}

All data treatments were performed using Origin Pro, Version 2018 (OriginLab Corporation, Northampton, MA, USA). In particular, a 3D spline was used to calculate the Ag density surface function: considering that this is a strictly interpolating function, a 2-nearest neighbors averaging was performed to significantly reduce the spline oscillations. The volume integral of the fitted surface was calculated using the same software.

A standard reference material of the National Institute of Standards and Technology (NIST SRM 610, certified Ag concentration $=268 \pm 29 \mathrm{mg} / \mathrm{kg}$ [44]) was used as drift correction standard to correct any variation of ICP-MS sensitivity and laser fluence. A $1600 \mu \mathrm{m}$ line was ablated using (i) a scan speed of $50 \mu \mathrm{m} / \mathrm{s}$, (ii) a spot size of $20 \mu \mathrm{m}$, (iii) a frequency of $20 \mathrm{~Hz}$ and (iv) a laser fluence of $3.6 \mathrm{~J} / \mathrm{cm}^{2}$. Such analysis was performed prior and after each grid of spots for sample analysis.

\subsection{Ag Dissolution and ICP-MS Analysis}

An ICP-MS, model Thermo Scientific ICAP Q, was used also for the mass spectrometric measurements of Ag extraction solution. The operating parameters of ICP-MS for all the measurements are the same as the ones reported in Table 1, except for the makeup gas flux, which is replaced by the nebulizer gas flux $(0.93 \mathrm{~L} / \mathrm{min})$.

The extraction solutions analyzed by ICP-MS were obtained only for "10 s" samples as follows:

Extraction \#1: After the first LA-ICP-MS analysis, a "10 s as-deposited" sample was treated with $2 \mathrm{~mL}$ of ultrapure nitric acid (obtained by sub-boiling distillation [45]) under sonication at room temperature for $2 \mathrm{~h}$. The polymeric disk was removed from the extraction solution and rinsed with ultrapure water (produced by a Sartorius Arium mini-UV lab water system). All rinsing solutions were added to the extraction solution and diluted with ultrapure water prior the ICP-MS analysis.

Extraction \#2: The polymeric sample resulting from extraction \#1 (labeled "10 s extr.\#1"), after a second LA-ICP-MS analysis, was treated in $2 \mathrm{~mL}$ of ultrapure nitric acid under sonication at $40{ }^{\circ} \mathrm{C}$ for $6 \mathrm{~h}$. The LDPE disk after extraction \#2 is labeled "10 s extr.\#2" and it was analyzed a third time by LA-ICP-MS.

Total Ag Extraction: A "10 s as-deposited" sample was treated with $2 \mathrm{~mL}$ of ultrapure $\mathrm{HNO}_{3}$ at $180{ }^{\circ} \mathrm{C}$ for $20 \mathrm{~min}$ in an ETHOS One (Milestone MLS) microwave digestion system equipped with home-made vessel-inside-vessel reactors (see more detail in [46,47]). This double container strategy, instead of putting the disk directly inside a single polytetrafluoroethylene (PTFE) microwave vessel, was adopted to avoid any possible Ag loss caused by $\mathrm{AgCl}$ precipitation [48]. After this extraction the LPDE disk was analyzed by LA-ICP-MS: 
no Ag signals were detected in the central region of the disk, confirming that Ag was totally extracted.

\section{Results}

\subsection{Characterization of Ag-Loaded Disks}

LDPE disks loaded with sub-microgram amounts of Ag NPs were chosen as model NP-loaded substrates for this study. Silver NPs were selected owing to their very wide application in the construction of supported functional materials, e.g., antibacterial and antifungal systems [49,50], sensing devices [51,52] and catalysts [53,54]. Ag nanoparticles are additionally the metallic NPs industrially produced in the largest amount [55]. A PMCS-SCBD was used to deposit Ag NPs because the radial anisotropic deposition may be tuned by simply increasing the Ar partial pressure in the deposition chamber: a highly focused deposition with Ag NPs deposited in less than $1 \mathrm{~mm}^{2}$ is achieved under high vacuum, whereas an increasingly spread deposition is obtained when the Ar pressure is raised as a result of the increasing number of collisions between Ar and the Ag NPs. A partial pressure equal to 17.3 Pa was selected in the present study, together with a target distance equal to $40 \mathrm{~mm}$, as these parameters lead to NPs being deposited onto a surface that is slightly lower than the target surface (see Experimental Section for further details).

Figure 2 reports the optical, SEM and HR-TEM characterization of the LDPE supported NPs deposited after a $10 \mathrm{~s}$ sputtering. Figure 2a (optical image) macroscopically confirms the expected spatial distribution: the central zone of the disk is visibly darker, suggesting that a much higher concentration of $\mathrm{Ag}$ is present there.
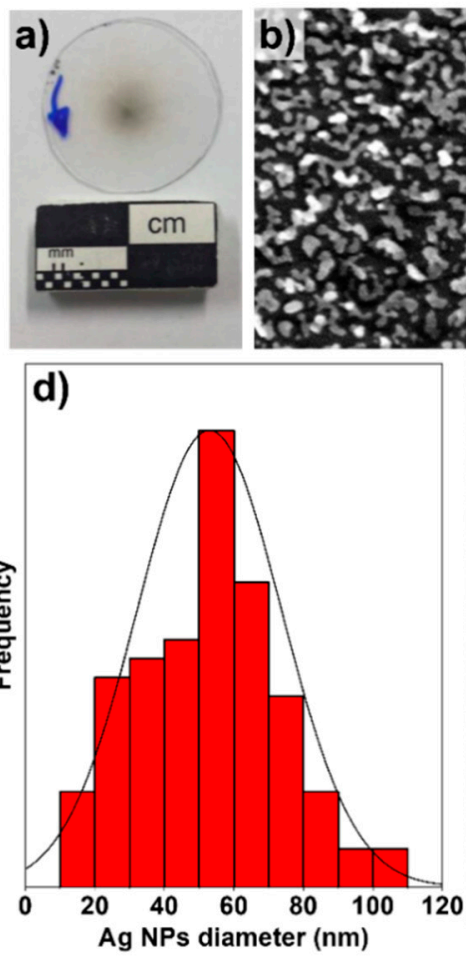

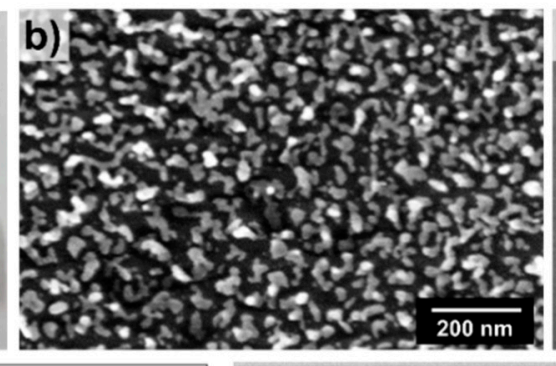

e)
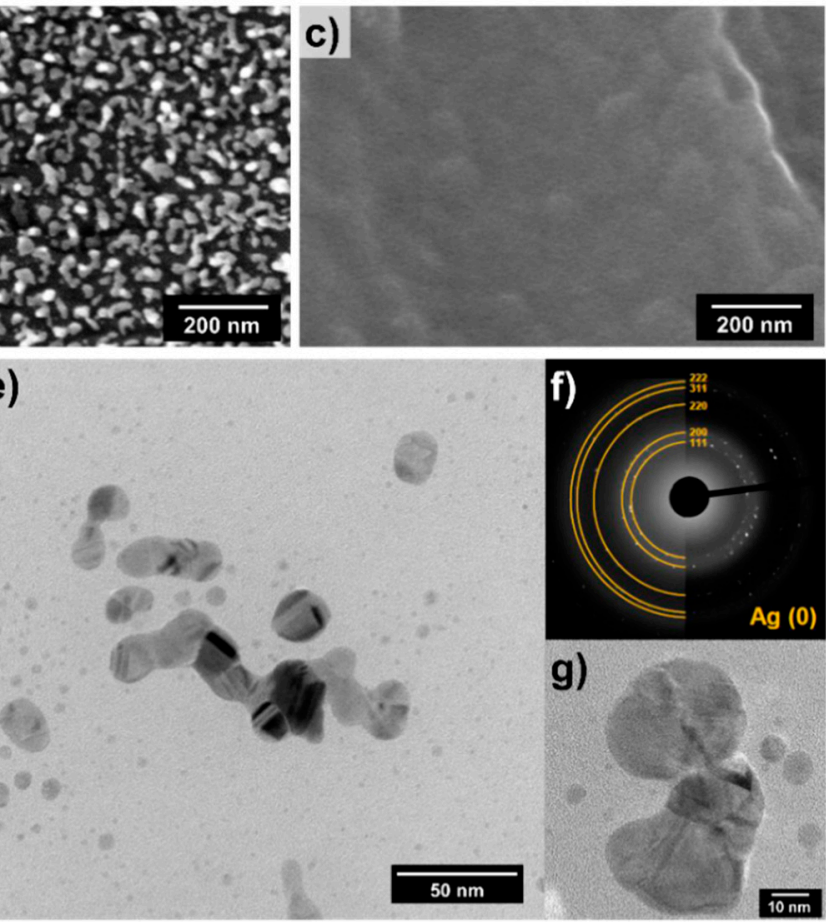

g)

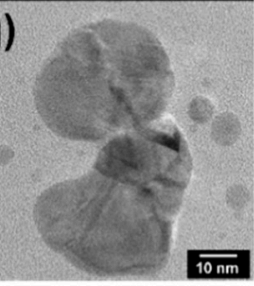

Figure 2. (a) Photo and (b) SEM top-view image of a "10 as-deposited" sample. (c) SEM top-view of a non-decorated LDPE disk. (d) Size distribution of Ag NPs in a "10 as-deposited" sample. (e,g) HR-TEM images and (f) electron diffraction image of Ag NPs.

The high density of Ag NPs in the center of the LDPE disk was also confirmed by SEM analysis (see Figure $2 b$ and, for the sake of comparison, see also a clean LDPE substrate in Figure 2c). As shown in Figure 2d, metal NPs exhibit a broad size distribution in the range 10-120 nm. HR-TEM analysis is in good agreement with the size distribution determined by SEM but it evidenced also the presence of NPs with a diameter lower than $10 \mathrm{~nm}$ (see 
Figure 2e,g). Finally, a good degree of crystallinity was observed by analyzing the electron diffraction pattern (Figure 2f), in which it is possible to observe the (111), (200), (220), (311) and (222) reflections of $\mathrm{Ag}$.

This system is accordingly a very good model of an anisotropic distribution of metal NPs with a noticeable trace concentration gradient over a substrate with macroscopic dimensions.

\subsection{Setup of the Calibration Protocol}

A viscous film sample chamber [36] was used in this work to achieve high sensitivity, needed to investigate even low amounts of $\mathrm{Ag}$, and fast analysis time over a quite large surface (disk area $\sim 3 \mathrm{~cm}^{2}$ ). In addition, it was demonstrated in previous work that signal intensities do not depend on the $X, Y$ coordinates of the ablated point [36]; i.e., a uniform sensitivity is expected over the entire sample surface. Multiple samples may be positioned inside this chamber, allowing the use of a drift correction standard to correct the timedependent drift of sensitivity. We analyzed the target analyte (Ag) in NIST SRM 610 prior and after each grid or line of spots, and a linear interpolation of the resulting signal with time was used to correct variations in sensitivity, as reported in previous papers [56-58].

A very simple cross design laser ablation pattern was initially adopted to gain preliminary insights about the Ag distribution on our model samples (see Figure 3a, 39 spots per line with $500 \mu \mathrm{m}$ spacing).

a)
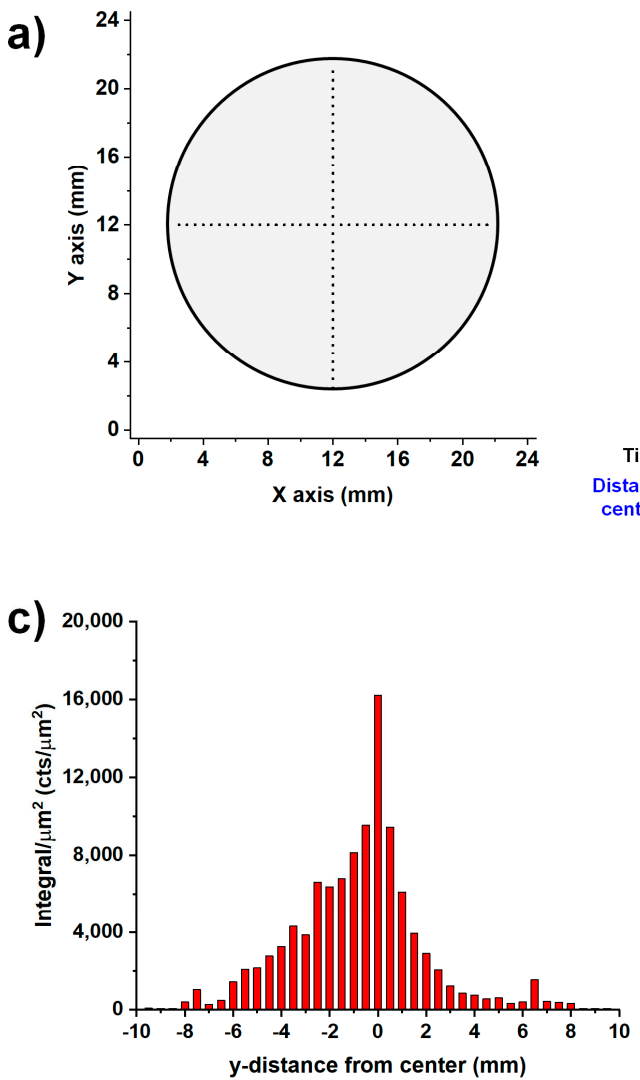

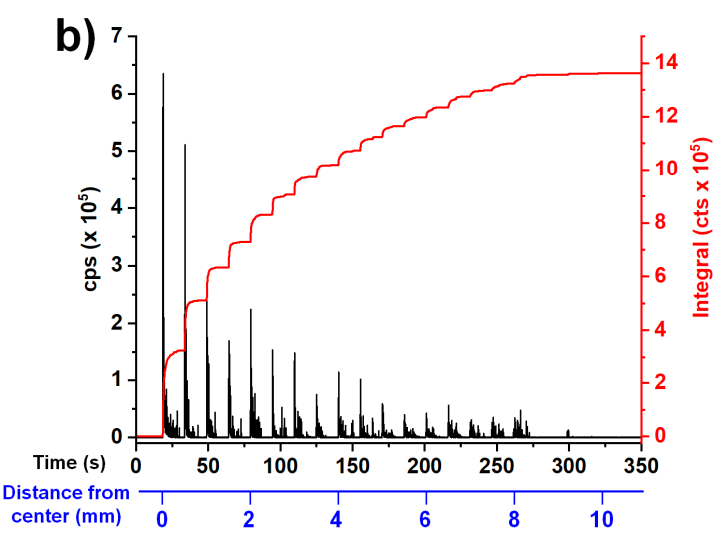

d)

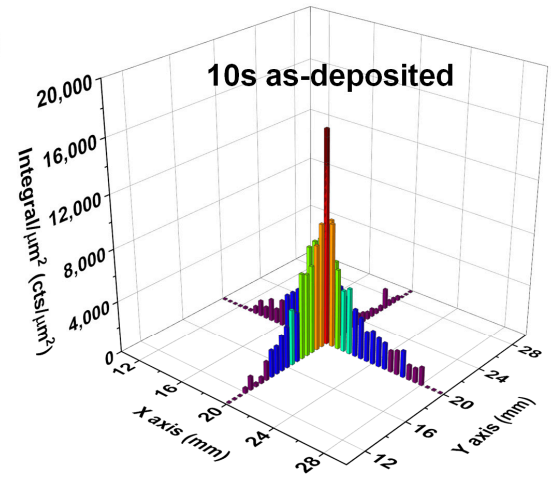

Figure 3. (a) First sampling pattern used to analyze the "10 s as-deposited" sample. The grayed circle represents the disk dimension. (b) LA-ICP-MS peaks and corresponding cumulative integral plot relative to the analysis of a line of 19 spots starting from the center of the disk (see experimental conditions in Table 1). (c) 2D bar and (d) 3D bar plot representation of the integral values found for each spot analysis (integral values were corrected according to the drift correction standard analysis and then divided by the laser spot size). 
The ICP-MS signals obtained with such an ablation pattern are depicted in Figure 3b, while the integrated signals are used to build the bar plots reported in Figure 3c,d. Please note that since instrumental signals are reported in counts-per-second (cps), the corresponding integrals are correctly reported as counts (cts). It is clearly visible that the Ag surface loading gradually decreases when moving away from the center of the disk. Nevertheless, the material ablated in the peripheral part of the disk is sufficient to generate an appreciable ICP-MS signal, meaning that the high sensitivity of LA-ICP-MS is fundamental to accurately describe the regions of the sample surface where very low amounts of NPs are expected. This model material is accordingly suited for the definition of the calibration protocol, namely, how to relate the ablation signals to the mass concentration per unit surface area.

We tackled this issue by applying two sequential processes. The first one relies on the definition of a suitable three-dimensional function which is used to fit the LA-ICP-MS integrals and thus to describe an Ag density 3D surface. The second part implies the determination of the Ag deposited mass on the polymeric disk (Ag extraction followed by ICP-MS analysis). The quantitative calibration is then obtained by linearly correlating the volume integral with the amount of extracted Ag. This calibration is carried out on a sacrificial sample but following quantifications can be performed by measuring the volume integral values and applying the calibration function without requiring Ag dissolution or other destructive techniques.

With respect to the development of a suitable fitting strategy, the integration of the peaks resulting from the two lines of spots (see Figure $3 c, d$ ) shows that there is no obvious analytical function that may describe NP distribution. We decided to use a 3D spline interpolation function (available in standard data manipulation software), because it is suitable to describe any NP distribution, making it the most general approach with widespread application.

This first screening highlighted that a different and more dense sampling scheme was needed to accurately describe the high Ag concentration gradient in the central zone of the disk. Accordingly, a grid of $17 \times 17$ spots was used covering a $4 \times 4 \mathrm{~mm}$ square in the central zone of the disk (spaced every $250 \mu \mathrm{m}$ ) plus 92 spots placed in 8 radial branches (500 $\mu \mathrm{m}$ spacing for horizontal and vertical lines, $707 \mu \mathrm{m}$ spacing for diagonal lines). This scheme is reported in Figure 4. It should be underlined that even using 381 spots, only a negligible portion of the entire surface is ablated (about $0.24 \%$ ).

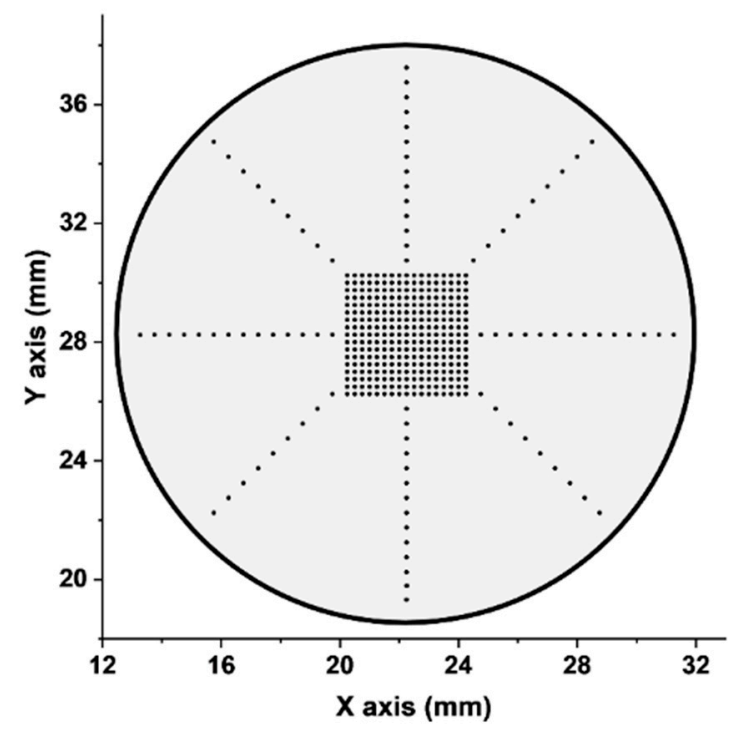

Figure 4. Revised sampling pattern consisting of 381 spots (grid $17 \times 17+4$ lines composed of 14 spots +4 diagonal lines composed of 9 spots). The grayed circle represents the disk dimension. 
The spatial distribution of the Ag integrated signals of the "10 s as-deposited" sample, together with the 3D spline reconstructed surface, are reported in Figure 5a,b, respectively, and the volume integral of the surface in Figure $5 b$ is reported in Table 2 (first row). The calibration of the signal was achieved by determining the Ag mass by the extraction of silver, followed by ICP-MS analysis. Mild conditions (see experimental section) were intentionally employed to dissolve Ag only partially: such conditions provide two calibration points from a single disk (see below). The analysis of the extraction solution revealed an Ag mass equal to $0.57 \mu \mathrm{g}$.

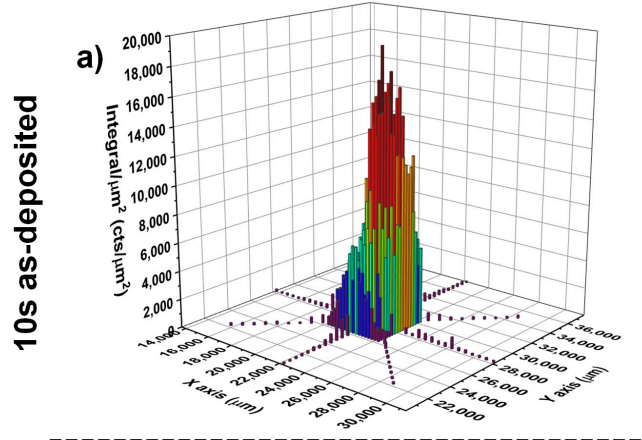

b)
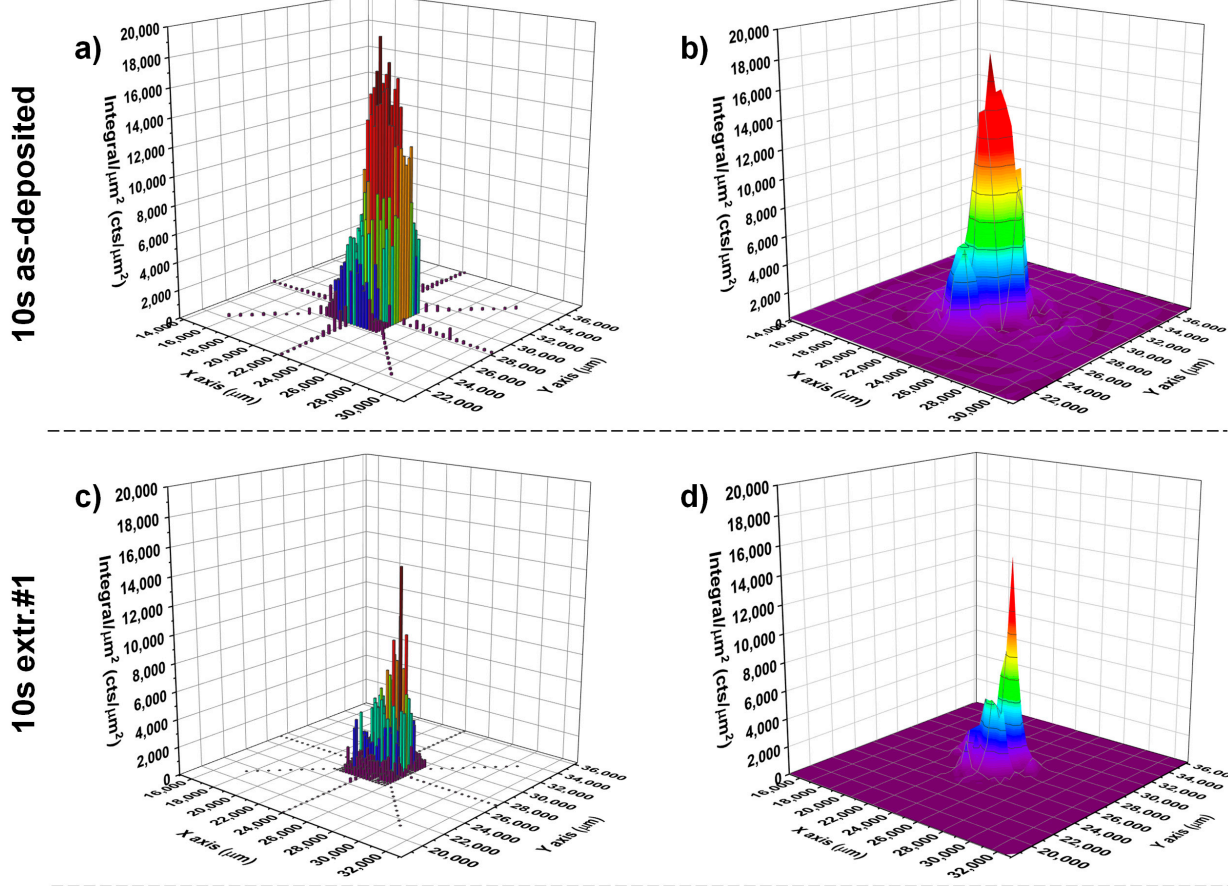

d)
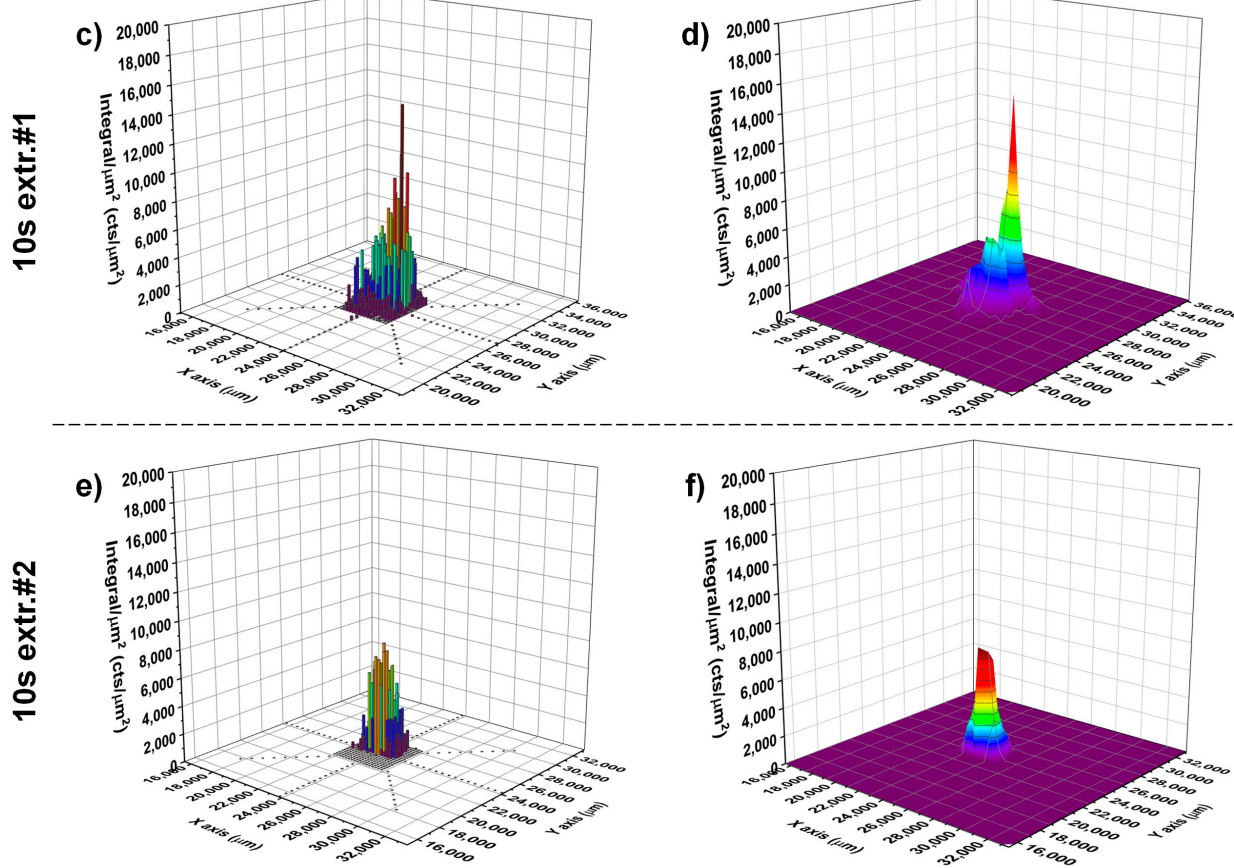

Figure 5. 3D bar plot and interpolated surface distribution of the integrated Ag signals for (a,b) "10 s as-deposited", (c,d) "10 s extr.\#1" and (e,f) "10 s extr.\#2".

Table 2. Summary of the results obtained by LA-ICP-MS and ICP-MS analysis.

\begin{tabular}{cccc}
\hline Sample & $\begin{array}{c}\text { Volume Integral of } \\
\text { Interpolated Surface (cts) }\end{array}$ & $\begin{array}{c}\text { Volume Integral } \\
\text { Difference (cts) }\end{array}$ & $\begin{array}{c}\text { Extracted Ag Mass } \\
(\boldsymbol{\mu g})\end{array}$ \\
\hline 10 s as-deposited & $1.91 \times 10^{11}$ & 0 & 0 \\
\hline 10 s extr.\#1 & $4.74 \times 10^{10}$ & $1.43 \times 10^{11}$ & $0.57 \pm 0.010$ \\
\hline 10 s extr.\#2 & $1.73 \times 10^{10}$ & $3.02 \times 10^{10}$ & $0.11 \pm 0.004$ \\
\hline
\end{tabular}

A second LA-ICP-MS analysis of the disk (labeled as "10 s extr.\#1") was performed, using the same pattern shown in Figure 4 (only $15 \mu \mathrm{m}$-shifted in both $\mathrm{X}$ and $\mathrm{Y}$ directions): 
the resulting data (see Figure 5c) were treated as shown for the pristine disk, thus obtaining a 3D spline reconstructed surface (Figure 5d) and its volume integral (Table 2, 2nd row). The volume integral of the fitted function is around four times lower than the one calculated for "10 s as-deposited", and the difference between these two volume integrals can be correlated to the amount of extracted Ag. Thus, the calibration factor can be easily obtained as the ratio between the difference of the volume integral values $\left(1.43 \times 10^{11} \mathrm{cts}\right)$ and the extracted Ag mass $(0.57 \mu \mathrm{g})$, which yields $2.51 \times 10^{11} \mathrm{cts} / \mu \mathrm{g}$. This sensitivity is the result of a one-point calibration.

An additional sequence of extraction/Ag determination/LA-ICP-MS analysis over the "10 s extr.\#1" sample was performed to check for the validity and the consistency of the proposed calibration procedure. This second extraction was performed in slightly harsher conditions in an attempt to dissolve a large portion of the residual Ag (see experimental section for details). The mass of Ag resulting from this second extraction was equal to $0.11 \mu \mathrm{g}$, while the 3D spline surface and its volume integral are reported in Figure $5 \mathrm{f}$ and Table 2 (3rd row), respectively.

The data reported in Table 2 were plotted to obtain the multipoint calibration curve reported in Figure 6. This figure clearly shows that there is a very good direct proportionality relationship between the volume integrals and the Ag mass on the surface.

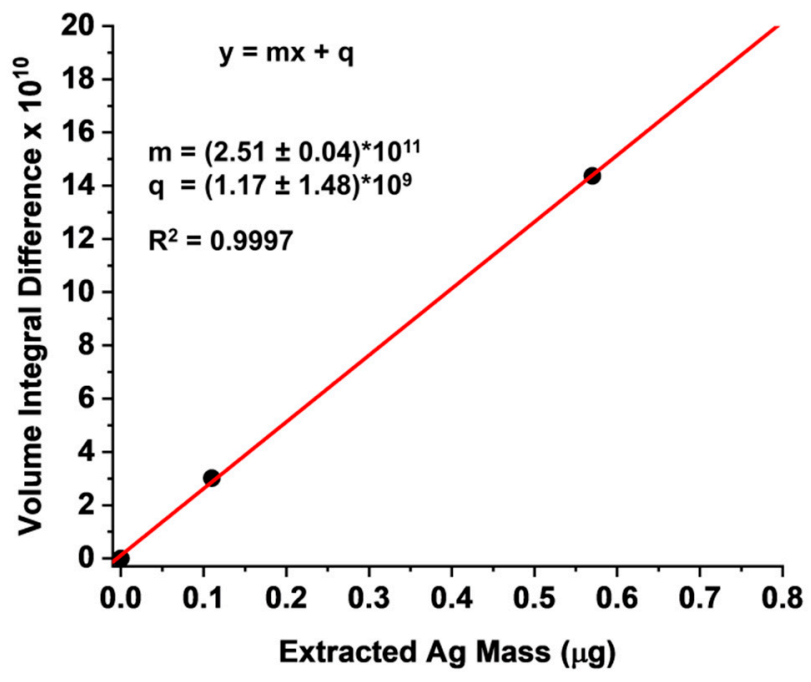

Figure 6. Calibration line obtained from data reported in Table 2.

\subsection{Validation of the Calibration Protocol}

The calibration procedure devised in the previous section was validated by applying the calibration reported in Figure 6 to another freshly prepared "10 s as-deposited" sample and comparing the result to the Ag mass obtained by ICP-MS after complete Ag dissolution (see experimental section for details). The very good agreement between these two datasets ( 0.76 and $0.78 \mu \mathrm{g}$ of Ag by LA-ICP-MS and solution analysis, respectively), indicates that this calibration protocol produces reliable data. This validation procedure is the only option as no standard reference material (SRM) for supported Ag NPs is available.

To further validate this protocol, two additional test samples were analyzed by LAICP-MS: (i) a "5 s as-deposited" sample was obtained exactly as the "10 s as-deposited", sputtering Ag for 5 s only; (ii) a " 5 s incorporated" was prepared as " $5 \mathrm{~s}$ as-deposited" but treating with dichloromethane vapors to induce a deeper penetration of Ag NPs into the polymeric matrix (see experimental section for details). Both samples were analyzed using the sampling pattern shown in Figure 4 and the described optimized data handling. Threedimensional spline surfaces and the results of the LA-ICP-MS quantifications are reported in Figure 7 and Table 3, respectively. Considering that the PMCS-SCBD method (used for Ag disks preparation) deposits the same amount of Ag in every pulse, the Ag loadings for the $5 \mathrm{~s}$ samples (15 pulses) were expected to be half of the ones obtained for the $10 \mathrm{~s}$ 
samples (30 pulses). The data are accordingly highly consistent (see Table 3), if compared to the $0.76 \mu \mathrm{g}$ of Ag determined for the "10 s as-deposited" sample, further supporting the adopted calibration procedure. Additionally, the total amount of Ag determined for the " 5 s incorporated" sample is comparable to the one determined for " $5 s$ as-deposited". In other words, the deeper penetration of Ag NPs into the polymer did not compromise the accuracy of the results achieved by LA-ICP-MS analysis.
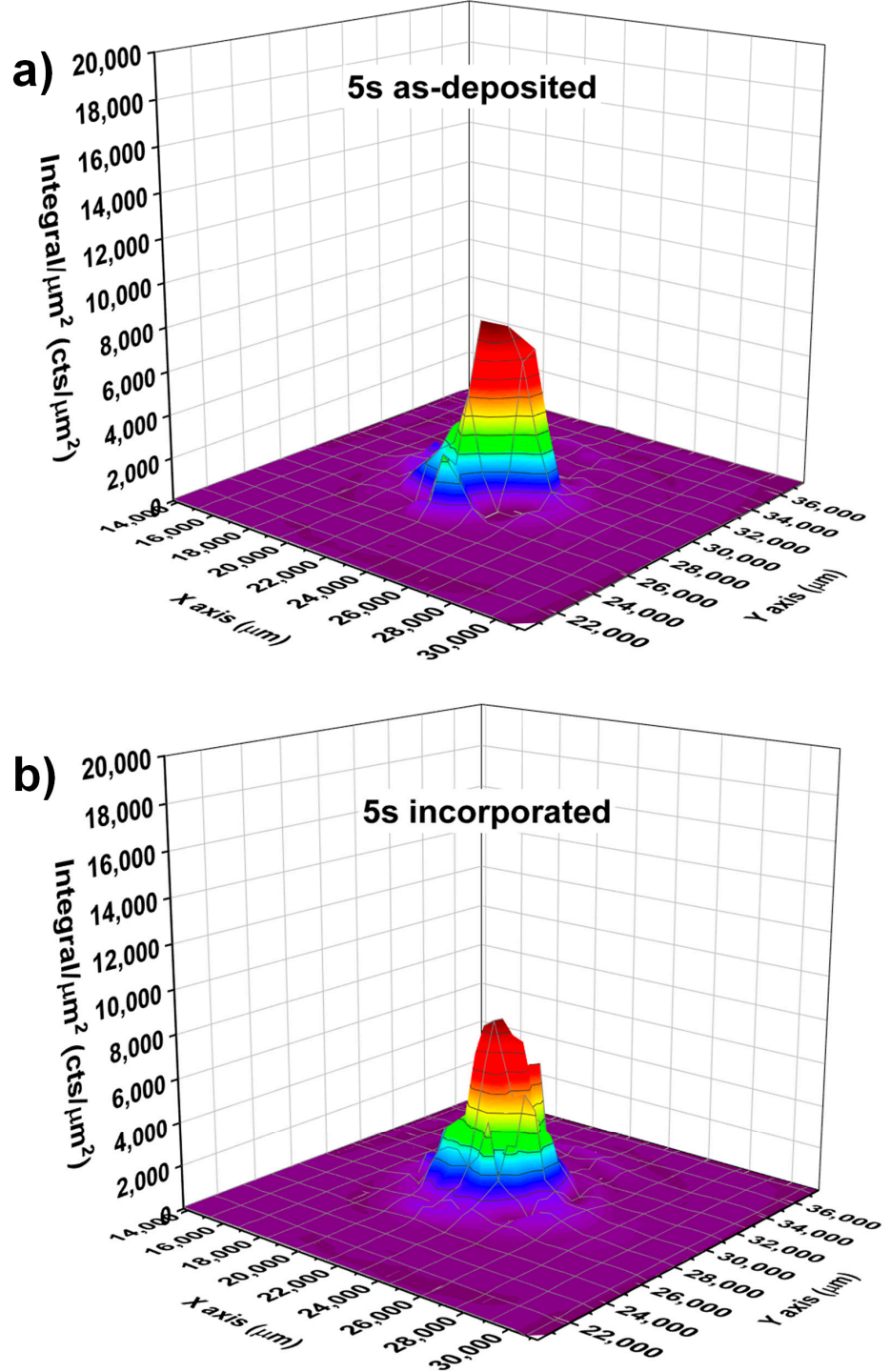

Figure 7. (a) Interpolated surface distribution for "5s as-deposited". (b) Interpolated surface distribution for " 5 s incorporated".

Table 3. Summary of Ag quantification performed only by LA-ICP-MS analysis on the " $5 \mathrm{~s}$ " samples.

\begin{tabular}{ccc}
\hline Sample & $\begin{array}{c}\text { Volume Integral of } \\
\text { Interpolated Surface (cts) }\end{array}$ & Calculated Ag Mass ( $\mu \mathrm{g})$ \\
\hline 5 s as-deposited & $1.01 \times 10^{11}$ & 0.40 \\
\hline 5 s incorporated & $9.40 \times 10^{10}$ & 0.37 \\
\hline
\end{tabular}

Finally, it should be underlined that the consistency of all the data so far reported allowed us to estimate the amount of Ag that the PMCS-SCBD method is able to produce, corresponding to a deposition rate of $25.6 \mathrm{ng} /$ pulse or $76.8 \mathrm{ng} / \mathrm{s}$. 


\subsection{Choice of the Ablation Pattern}

The quantitative results so far presented were obtained with the ablation pattern reported in Figure 4. This pattern was prudently dense in the central part of the surface where the gradient in NP concentration was higher. To critically evaluate if it was actually necessary to use such a huge number of ablation points (381), we calculated the results that can be obtained by two subsequent halvings of the original dataset, i.e., using 190 and 95 points to build the 3D surface. The data reported in Table 4 show that, even with the smallest dataset, the total amount of $\mathrm{Ag}$ was correctly determined. The reduction of the dataset had strongly beneficial effects in terms of analysis time, since the latter was directly proportional to the number of ablated spots.

Table 4. Ag quantification performed by LA-ICP-MS analysis by changing the number of ablated spots.

\begin{tabular}{ccccc}
\hline Sample & $\begin{array}{c}\text { Number of Ablated } \\
\text { Spots }\end{array}$ & $\begin{array}{c}\text { Required Analysis } \\
\text { Time (min) }\end{array}$ & $\begin{array}{c}\text { Volume Integral of } \\
\text { Interpolated Surface (cts) }\end{array}$ & $\begin{array}{c}\text { Calculated Ag Mass } \\
(\mu \mathrm{g})\end{array}$ \\
\hline 10 s as-deposited-1 & 381 & $\sim 100$ & $1.91 \times 10^{11}$ & 0.76 \\
\hline 10 s as-deposited-2 & 190 & $\sim 50$ & $1.87 \times 10^{11}$ & 0.75 \\
\hline 10 s as-deposited-3 & 95 & $\sim 25$ & $1.91 \times 10^{11}$ & 0.76 \\
\hline
\end{tabular}

However, the decrease in the sampling points may lead to a poorer description of the Ag distribution, as may be noticed by spotting; e.g., the fine detail (at 23 and $26 \mathrm{~mm}$ ) that seemed to disappear as the sampling frequency decreased (see Figure 8).
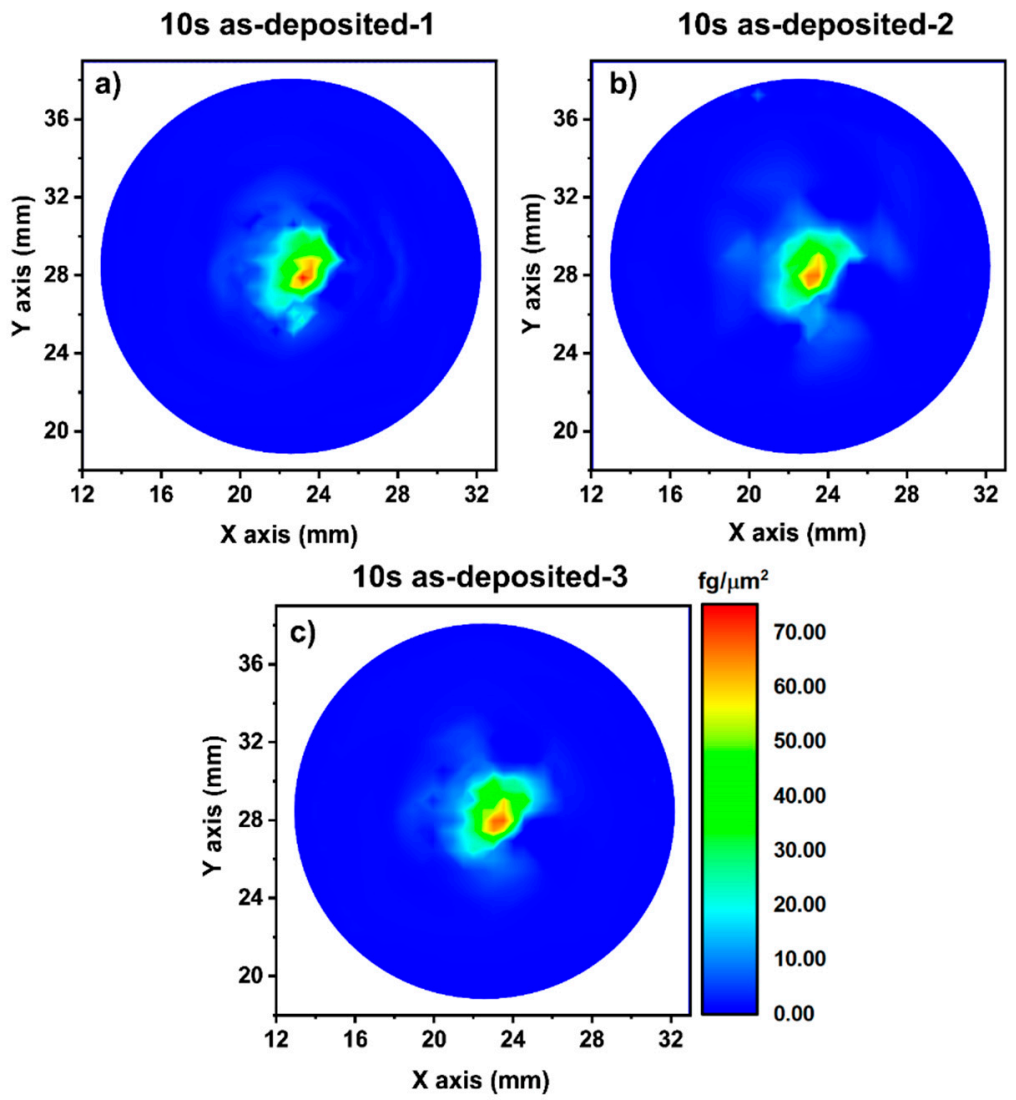

Figure 8. Ag mass contour distribution map obtained for the "10 s as-deposited" with (a) 381 points, (b) 190 points and (c) 95 points.

The distribution and the number of points to be used in the ablation pattern should be evaluated case by case, as they strictly depend on the shape and on the smoothness of the 
distribution. The best ablation pattern also depends on the sought information, namely the total metal loading vs. the highly or limitedly detailed description of the metal distribution.

\section{Conclusions}

An LA-ICP-MS-based approach for the quantitative estimation of the spatial distribution of metal NPs deposited on solid supports was developed. We demonstrated that, thanks to a proper sampling pattern and data elaboration, the determination of both the total deposited mass and its spatial distribution can be achieved using a micro-destructive analytical technique, as only $0.24 \%$ of the total surface is ablated. Trace and ultratrace levels of NPs, down to the $\mathrm{fg} / \mu \mathrm{m}^{2}$ level, may be determined by this analytical protocol, as required when very low metal loadings are used, or areas interested by marginal deposition are studied. The method also allows the determination of the absolute loading of any given portion of the sample surface by simply calculating the volume integral of the 3D spline limited by the surface of interest.

Although only the distribution of silver NPs on LDPE was determined in the present work, the extension to any metal-loaded surface may be easily foreseen, as laser ablation ensures an efficient vaporization of almost any material, whereas ICP-MS is a universal trace detector for all the metallic elements. The use of the 3D spline interpolation further contributes to the vast applicability of the procedure as this interpolating function does not require any a priori knowledge about the NPs' distribution. Nevertheless, a preliminary investigation may help in defining a time efficient sampling pattern, whose geometry and frequency should be set as a function of the shape and the smoothness of the anisotropic distribution, respectively.

The potential applicability of the present approach is surely wide, as it may find employment in all applications where anisotropically modified surfaces are investigated or the homogeneity of NP deposition needs to be verified. Application to substrates other than polymers seems at hand since surface NPs are easily removed by the laser beam irrespective of the support, although a dedicated calibration procedure may be possibly required. Possible applications thus include the characterization of supported catalysts, nanostructured electrodes and, more generally, sensing devices. Finally, as reported in the present work, the proposed protocol may also be used to assess the amount of deposited NPs and the deposition profile generated by any kind of nanoparticle source.

Author Contributions: Conceptualization, D.M. and S.R.; methodology, D.M.; investigation, D.S., G.B. and M.M.; data curation, D.S. and G.B.; writing-original draft preparation, D.S. and S.R.; writing-review and editing, D.M. and L.R.; visualization, D.S. and G.B.; supervision, D.M. and S.R. All authors have read and agreed to the published version of the manuscript.

Funding: This research received no external funding.

Institutional Review Board Statement: Not applicable.

Informed Consent Statement: Not applicable.

Data Availability Statement: Not applicable.

Conflicts of Interest: The authors declare no conflict of interest.

\section{References}

1. Ndolomingo, M.J.; Bingwa, N.; Meijboom, R. Review of supported metal nanoparticles: Synthesis methodologies, advantages and application as catalysts. J. Mater. Sci. 2020, 55, 6195-6241. [CrossRef]

2. Liu, L.; Corma, A. Metal Catalysts for Heterogeneous Catalysis: From Single Atoms to Nanoclusters and Nanoparticles. Chem. Rev. 2018, 118, 4981-5079. [CrossRef]

3. Campelo, J.M.; Luna, D.; Luque, R.; Marinas, J.M.; Romero, A.A. Sustainable Preparation of Supported Metal Nanoparticles and Their Applications in Catalysis. ChemSusChem 2009, 2, 18-45. [CrossRef]

4. Zou, X.; Wang, J.; Liu, X.; Wang, C.; Jiang, Y.; Wang, Y.; Xiao, X.; Ho, J.C.; Li, J.; Jiang, C.; et al. Rational Design of Sub-Parts per Million Specific Gas Sensors Array Based on Metal Nanoparticles Decorated Nanowire Enhancement-Mode Transistors. Nano Lett. 2013, 13, 3287-3292. [CrossRef] [PubMed] 
5. Gutés, A.; Hsia, B.; Sussman, A.; Mickelson, W.; Zettl, A.; Carraro, C.; Maboudian, R. Graphene decoration with metal nanoparticles: Towards easy integration for sensing applications. Nanoscale 2012, 4, 438-440. [CrossRef]

6. Spanu, D.; Binda, G.; Dossi, C.; Monticelli, D. Biochar as an alternative sustainable platform for sensing applications: A review. Microchem. J. 2020, 159, 105506. [CrossRef]

7. Nava, G.; Fumagalli, F.; Gambino, S.; Farella, I.; Dell'Erba, G.; Beretta, D.; Divitini, G.; Ducati, C.; Caironi, M.; Cola, A.; et al Towards an electronic grade nanoparticle-assembled silicon thin film by ballistic deposition at room temperature: The deposition method, and structural and electronic properties. J. Mater. Chem. C 2017, 5, 3725-3735. [CrossRef]

8. Ko, S.H.; Park, I.; Pan, H.; Grigoropoulos, C.P.; Pisano, A.P.; Luscombe, C.K.; Fréchet, J.M.J. Direct Nanoimprinting of Metal Nanoparticles for Nanoscale Electronics Fabrication. Nano Lett. 2007, 7, 1869-1877. [CrossRef] [PubMed]

9. Kelly, P.; Arnell, R. Magnetron sputtering: A review of recent developments and applications. Vacuum 2000, 56, 159-172. [CrossRef]

10. Spanu, D.; Bestetti, A.; Hildebrand, H.; Schmuki, P.; Altomare, M.; Recchia, S. Photocatalytic reduction and scavenging of Hg(II) over templated-dewetted $\mathrm{Au}$ on $\mathrm{TiO}_{2}$ nanotubes. Photochem. Photobiol. Sci. 2019, 18, 1046-1055. [CrossRef] [PubMed]

11. Voevodin, A.A.; Donley, M.S.; Zabinski, J.S. Pulsed laser deposition of diamond-like carbon wear protective coatings: A review. Surf. Coat. Technol. 1997, 92, 42-49. [CrossRef]

12. Lorenz, M.; Ramachandra Rao, M.S. 25 years of pulsed laser deposition. J. Phys. D Appl. Phys. 2014, 47, 030301. [CrossRef]

13. Wenderich, K.; Mul, G. Methods, Mechanism, and Applications of Photodeposition in Photocatalysis: A Review. Chem. Rev. 2016, 116, 14587-14619. [CrossRef]

14. Huang, L.; Liu, X.; Wu, H.; Wang, X.; Wu, H.; Li, R.; Shi, L.; Li, C. Surface state modulation for size-controllable photodeposition of noble metal nanoparticles on semiconductors. J. Mater. Chem. A 2020, 8, 21094-21102. [CrossRef]

15. Mohanty, U.S. Electrodeposition: A versatile and inexpensive tool for the synthesis of nanoparticles, nanorods, nanowires, and nanoclusters of metals. J. Appl. Electrochem. 2011, 41, 257-270. [CrossRef]

16. Quinn, B.M.; Dekker, C.; Lemay, S.G. Electrodeposition of Noble Metal Nanoparticles on Carbon Nanotubes. J. Am. Chem. Soc. 2005, 127, 6146-6147. [CrossRef]

17. Fu, X.; Yu, H.; Peng, F.; Wang, H.; Qian, Y. Facile preparation of RuO2/CNT catalyst by a homogenous oxidation precipitation method and its catalytic performance. Appl. Catal. A Gen. 2007, 321, 190-197. [CrossRef]

18. Spanu, D.; Dal Santo, V.; Malara, F.; Naldoni, A.; Turolla, A.; Antonelli, M.; Dossi, C.; Marelli, M.; Altomare, M.; Schmuki, P.; et al. Photoelectrocatalytic oxidation of As(III) over hematite photoanodes: A sensible indicator of the presence of highly reactive surface sites. Electrochim. Acta 2018, 292, 828-837. [CrossRef]

19. Chávez, F.; Pérez-Sánchez, G.F.; Goiz, O.; Zaca-Morán, P.; Peña-Sierra, R.; Morales-Acevedo, A.; Felipe, C.; Soledad-Priego, M. Sensing performance of palladium-functionalized WO3 nanowires by a drop-casting method. Appl. Surf. Sci. 2013, 275, 28-35. [CrossRef]

20. Torres-Rivero, K.; Torralba-Cadena, L.; Espriu-Gascon, A.; Casas, I.; Bastos-Arrieta, J.; Florido, A. Strategies for surface modification with ag-shaped nanoparticles: Electrocatalytic enhancement of screen-printed electrodes for the detection of heavy metals. Sensors 2019, 19, 4249. [CrossRef]

21. Xie, F.; Baker, M.S.; Goldys, E.M. Homogeneous Silver-Coated Nanoparticle Substrates for Enhanced Fluorescence Detection. J. Phys. Chem. B 2006, 110, 23085-23091. [CrossRef]

22. Cui, F.; Hua, Z.; Wei, C.; Li, J.; Gao, Z.; Shi, J. Highly dispersed Au nanoparticles incorporated mesoporous TiO2 thin films with ultrahigh Au content. J. Mater. Chem. 2009, 19, 7632. [CrossRef]

23. Zhang, X.B.; Pei, Z.L.; Gong, J.; Sun, C. Investigation on the electrical properties and inhomogeneous distribution of ZnO:Al thin films prepared by dc magnetron sputtering at low deposition temperature. J. Appl. Phys. 2007, 101, 014910. [CrossRef]

24. Czajkowski, K.M.; Świtlik, D.; Langhammer, C.; Antosiewicz, T.J. Effective Optical Properties of Inhomogeneously Distributed Nanoobjects in Strong Field Gradients of Nanoplasmonic Sensors. Plasmonics 2018, 13, 2423-2434. [CrossRef] [PubMed]

25. Ianoul, A.; Bergeron, A. Spatially Inhomogeneous Enhancement of Fluorescence by a Monolayer of Silver Nanoparticles. Langmuir 2006, 22, 10217-10222. [CrossRef]

26. Newbury, D.E.; Ritchie, N.W.M. Is Scanning Electron Microscopy/Energy Dispersive X-ray Spectrometry (SEM/EDS) Quantitative? Scanning 2013, 35, 141-168. [CrossRef]

27. de Moura Souza, F.; Pollo Paniz, F.; Pedron, T.; Coelho dos Santos, M.; Lemos Batista, B. A high-throughput analytical tool for quantification of 15 metallic nanoparticles supported on carbon black. Heliyon 2019, 5, e01308. [CrossRef]

28. Bland, G.D.; Lowry, G.V. Multistep Method to Extract Moderately Soluble Copper Oxide Nanoparticles from Soil for Quantification and Characterization. Anal. Chem. 2020, 92, 9620-9628. [CrossRef]

29. Wajid, A. On the accuracy of the quartz-crystal microbalance (QCM) in thin-film depositions. Sens. Actuators A Phys. 1997, 63, 41-46. [CrossRef]

30. Snook, G.A.; Chen, G.Z.; Fray, D.J.; Hughes, M.; Shaffer, M. Studies of deposition of and charge storage in polypyrrole-chloride and polypyrrole-carbon nanotube composites with an electrochemical quartz crystal microbalance. J. Electroanal. Chem. 2004, 568, 135-142. [CrossRef]

31. Pozebon, D.; Scheffler, G.L.; Dressler, V.L. Recent applications of laser ablation inductively coupled plasma mass spectrometry (LA-ICP-MS) for biological sample analysis: A follow-up review. J. Anal. At. Spectrom. 2017, 32, 890-919. [CrossRef] 
32. Limbeck, A.; Galler, P.; Bonta, M.; Bauer, G.; Nischkauer, W.; Vanhaecke, F. Recent advances in quantitative LA-ICP-MS analysis: Challenges and solutions in the life sciences and environmental chemistry. Anal. Bioanal. Chem. 2015, 407, 6593-6617. [CrossRef]

33. Binda, G.; Di Iorio, A.; Monticelli, D. The what, how, why, and when of dendrochemistry: (paleo)environmental information from the chemical analysis of tree rings. Sci. Total Environ. 2021, 758, 143672. [CrossRef]

34. Chew, D.; Drost, K.; Marsh, J.H.; Petrus, J.A. LA-ICP-MS imaging in the geosciences and its applications to geochronology. Chem. Geol. 2021, 559, 119917. [CrossRef]

35. Giussani, B.; Monticelli, D.; Rampazzi, L. Role of laser ablation-inductively coupled plasma-mass spectrometry in cultural heritage research: A review. Anal. Chim. Acta 2009, 635, 6-21. [CrossRef] [PubMed]

36. Monticelli, D.; Civati, D.; Giussani, B.; Dossi, C.; Spanu, D.; Recchia, S. A viscous film sample chamber for Laser Ablation Inductively Coupled Plasma-Mass Spectrometry. Talanta 2018, 179, 100-106. [CrossRef]

37. Asogan, D.; Sharp, B.L.; Connor, C.J.P.O.; Green, D.A.; Hutchinson, R.W. An open, non-contact cell for laser ablation-inductively coupled plasma-mass spectrometry. J. Anal. At. Spectrom. 2009, 24, 917. [CrossRef]

38. Horak, F.; Nagl, A.; Föttinger, K.; Limbeck, A. Application of micro-dried droplets for quantitative analysis of particulate inorganic samples with LA-ICP-MS demonstrated on surface-modified nanoparticle $\mathrm{TiO}_{2}$ catalyst materials. Microchim. Acta 2020, 187,641 . [CrossRef]

39. Benešová, I.; Dlabková, K.; Zelenák, F.; Vaculovič, T.; Kanický, V.; Preisler, J. Direct Analysis of Gold Nanoparticles from Dried Droplets Using Substrate-Assisted Laser Desorption Single Particle-ICPMS. Anal. Chem. 2016, 88, 2576-2582. [CrossRef]

40. Wegner, K.; Piseri, P.; Tafreshi, H.V.; Milani, P. Cluster beam deposition: A tool for nanoscale science and technology. J. Phys. D Appl. Phys. 2006, 39, R439-R459. [CrossRef]

41. Barborini, E.; Conti, A.M.; Kholmanov, I.; Piseri, P.; Podestà, A.; Milani, P.; Cepek, C.; Sakho, O.; Macovez, R.; Sancrotti, M. Nanostructured TiO2 Films with 2 eV Optical Gap. Adv. Mater. 2005, 17, 1842-1846. [CrossRef]

42. Barborini, E.; Piseri, P.; Milani, P. A pulsed microplasma source of high intensity supersonic carbon cluster beams. J. Phys. D Appl. Phys. 1999, 32, L105-L109. [CrossRef]

43. Dossi, C.; Binda, G.; Monticelli, D.; Pozzi, A.; Recchia, S.; Spanu, D. Exploiting Laser-Ablation ICP-MS for the Characterization of Salt-Derived Bismuth Films on Screen-Printed Electrodes: A Preliminary Investigation. Biosensors 2020, 10, 119. [CrossRef]

44. Jochum, K.P.; Weis, U.; Stoll, B.; Kuzmin, D.; Yang, Q.; Raczek, I.; Jacob, D.E.; Stracke, A.; Birbaum, K.; Frick, D.A.; et al. Determination of Reference Values for NIST SRM 610-617 Glasses Following ISO Guidelines. Geostand. Geoanalytical Res. 2011, 35, 397-429. [CrossRef]

45. Monticelli, D.; Castelletti, A.; Civati, D.; Recchia, S.; Dossi, C. How to Efficiently Produce Ultrapure Acids. Int. J. Anal. Chem. 2019, 2019, 1-5. [CrossRef] [PubMed]

46. Spanu, D.; Butti, L.; Boldrocchi, G.; Bettinetti, R.; Monticelli, D. High-throughput, Multi-batch System for the Efficient Microwave Digestion of Biological Samples. Anal. Sci. 2020, 36, 889-892. [CrossRef] [PubMed]

47. Boldrocchi, G.; Monticelli, D.; Mazzoni, M.; Spanu, D.; Bettinetti, R. Accumulation of Selected Trace Elements in Shads from Three Lakes: First Insights from Italian Pre-Alpine Area. Biol. Trace Elem. Res. 2021. [CrossRef]

48. Recchia, S.; Spanu, D.; Bianchi, D.; Dossi, C.; Pozzi, A.; Monticelli, D. Understanding microwave vessel contamination by chloride species. Talanta 2016, 159. [CrossRef] [PubMed]

49. Fahmy, H.M.; Salah Eldin, R.E.; Abu Serea, E.S.; Gomaa, N.M.; AboElmagd, G.M.; Salem, S.A.; Elsayed, Z.A.; Edrees, A.; ShamsEldin, E.; Shalan, A.E. Advances in nanotechnology and antibacterial properties of biodegradable food packaging materials. RSC Adv. 2020, 10, 20467-20484. [CrossRef]

50. Cruz, M.C.; Ruano, G.; Wolf, M.; Hecker, D.; Castro Vidaurre, E.; Schmittgens, R.; Rajal, V.B. Plasma deposition of silver nanoparticles on ultrafiltration membranes: Antibacterial and anti-biofouling properties. Chem. Eng. Res. Des. 2015, 94, 524-537. [CrossRef]

51. Gupta, S.; Uhlmann, P.; Agrawal, M.; Chapuis, S.; Oertel, U.; Stamm, M. Immobilization of Silver Nanoparticles on Responsive Polymer Brushes. Macromolecules 2008, 41, 2874-2879. [CrossRef]

52. Niu, B.; Hua, T.; Xu, B. Robust Deposition of Silver Nanoparticles on Paper Assisted by Polydopamine for Green and Flexible Electrodes. ACS Sustain. Chem. Eng. 2020, 8, 12842-12851. [CrossRef]

53. Albiter, E.; Valenzuela, M.A.; Alfaro, S.; Valverde-Aguilar, G.; Martínez-Pallares, F.M. Photocatalytic deposition of Ag nanoparticles on TiO2: Metal precursor effect on the structural and photoactivity properties. J. Saudi Chem. Soc. 2015, 19, 563-573. [CrossRef]

54. Pagliaro, M.; Della Pina, C.; Mauriello, F.; Ciriminna, R. Catalysis with Silver: From Complexes and Nanoparticles to MORALs and Single-Atom Catalysts. Catalysts 2020, 10, 1343. [CrossRef]

55. Pulit-Prociak, J.; Banach, M. Silver nanoparticles-A material of the future ... ? Open Chem. 2016, 14, 76-91. [CrossRef]

56. Lin, J.; Liu, Y.; Yang, Y.; Hu, Z. Calibration and correction of LA-ICP-MS and LA-MC-ICP-MS analyses for element contents and isotopic ratios. Solid Earth Sci. 2016, 1, 5-27. [CrossRef]

57. Liu, Y.; Hu, Z.; Gao, S.; Günther, D.; Xu, J.; Gao, C.; Chen, H. In situ analysis of major and trace elements of anhydrous minerals by LA-ICP-MS without applying an internal standard. Chem. Geol. 2008, 257, 34-43. [CrossRef]

58. Liu, Y.; Hu, Z.; Li, M.; Gao, S. Applications of LA-ICP-MS in the elemental analyses of geological samples. Chin. Sci. Bull. 2013, 58, 3863-3878. [CrossRef] 